\title{
Arrhinia-choanal atresia-microphthalmia syndrome
}

INSERM

\section{Source}

INSERM. (1999). Orphanet: an online rare disease and orphan drug data base. Arrhiniachoanal atresia-microphthalmia syndrome. ORPHA:1135

Arhinia-choanal atresia-microphthalmia is a malformation disorder characterized by complete or incomplete absence of nose (arrhinia), choanal atresia, microphthalmia, anophthalmia and cleft or high palate. 